\title{
Single and double distributed optical amplifier fiber bus networks with wavelength-division multiplexing for photonic sensors
}

\author{
Silvia Abad and Manuel López-Amo \\ Departamento de Ingeniería Eléctrica y Electrónica, Universidad Pública de Navarra, Campus de Arrosadía s/n, 31006 Pamplona, Spain
}

Jose Miguel López-Higuera

Escuela Técnica Superior de Ingenieros Industriales y Telecomunicación, Grupo de Ingeniería Fontónica, Universidad de Cantabria, Avenida Los Castros s/n, 39005 Santander, Spain

David Benito, Amaya Unanua, and Elisa Achaerandio

Departamento de Ingeniería Eléctrica y Electrónica, Universidad Pública de Navarra, Campus de Arrosadía s/n, 31006 Pamplona, Spain

Received January 28, 1999

\begin{abstract}
Two different optical fiber bus networks are compared and demonstrated experimentally as means of gathering information from four wavelength-division-multiplexed photonic sensors. Both topologies include distributed amplification, which allows one to overcome the limitation in the maximum number of sensors that can be multiplexed in a single structure. Results obtained with a dual-bus topology are compared with those achieved with a single-bus network. (C) 1999 Optical Society of America
\end{abstract}

OCIS codes: $\quad 060.4230,060.2370,060.2410,060.2300,060.4250$.

The proliferation of the so-called smart materials that are employed in aeronautics, medical applications, and intelligent buildings is increasing the number of sensors that need to be dealt with in a single structure. It is in these fields that photonic sensors exhibit their advantages, especially their low weight and small size. Bus architectures are widely used in data and sensor networks because of their simple cabling requirements, especially when a large number of sensors (or stations) must be multiplexed. The main disadvantage of these topologies is the power loss that is introduced by the couplers, which limits the number of sensors that can be connected to the structure and causes a difference in the power that is received from each sensor, depending on the number of couplers that the signal must cross to reach it.

Overcoming the limitation imposed by the bus structures by use of optical amplification has been proposed $^{1}$ and has been demonstrated in both lumped ${ }^{2}$ and distributed $^{3}$ configurations. The utilization of amplifying fiber between couplers for this purpose is particularly appealing. Thus the gain of each segment of fiber counterbalances the attenuation that is introduced by the coupler located after it. This distributed amplification yields constant power in each sensor and a minor overall loss, allowing a greater number of sensors to be multiplexed in each structure. On the other hand, the use of erbium-doped fiber introduces an important source of noise, amplified spontaneous emission (ASE), that has to be accounted for.

In this Letter we demonstrate experimentally the single- and dual-bus structures shown in Fig. 1; simulations of these structures have already been published. ${ }^{4}$ The sensors that are used here provide amplitude modulation in response to the curvature introduced by a temperature-sensitive element. ${ }^{5}$ Each sensor is identified by a different wavelength, assigned by the fiber Bragg grating (FBG) located after it. The total insertion losses of each sensor and the FBG are $6 \mathrm{~dB}$. We locate the operation wavelengths of the FBG in the flat region of the erbium gain profile to provide good equalization among channels 1539.60, 1547.77, 1552.23 , and $1560.09 \mathrm{~nm}$. The FBG's bandwidths are approximately $0.5 \mathrm{~nm}$.

The signal is provided by a tunable laser source with an output power of $-10 \mathrm{dBm}$ and a $0.2-\mathrm{nm}$ linewidth. The pump scheme is a three-level diagram and uses a $980-\mathrm{nm}$ laser that delivers $60 \mathrm{~mW}$ of power into the bus. We select the pump power and the length

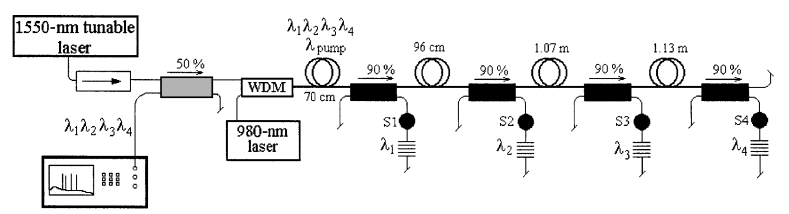

(a)

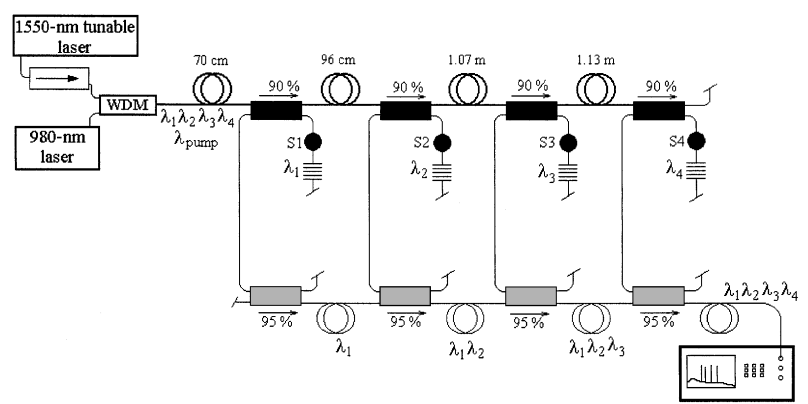

(b)

Fig. 1. (a) Wavelength-division-multiplexed optical fiber single-bus network with distributed gain for sensors. (b) Equivalent dual network, including a nonamplifying lower bus. WDM's, wavelength-division multiplexers; S1-S4, sensors.

(C) 1999 Optical Society of America 
of the active fiber segments to provide 1-dB gain, which approximately compensates for the loss from the following coupler. We immerse all the free terminations on the bus in refractive-index-matching gel to avoid unwanted reflections.

In the single topology [Fig. 1(a)] the same bus is used to carry both methods of propagation. The incoming power propagates from left to right, and the modulated signal travels from right to left. The 50:50 coupler on the header divides these two signals and introduces a 6-dB loss. If this loss is to be avoided, the coupler should be replaced with a circulator, although this solution would increase the complexity and the price of the system. In Fig. 2 the four return signals in the single configuration are superimposed upon the ASE pedestal. We obtained these results by applying the technique for allocating the signal wavelength to the sensor position to equalize the received signal power that was described in Ref. 4. The amount of noise in each channel, the signal power, and the signal-tonoise ratio (SNR) are shown in Table 1 . The average signal power is $\sim-41.5 \mathrm{dBm}$, with a difference of $1.7 \mathrm{~dB}$ between extreme channels. Had we used a passive structure, this difference would have been a value of $\sim 3.6 \mathrm{~dB}$ (taking into account a 0.6 - $\mathrm{dB}$ loss in each coupler). The SNR values are close to $13 \mathrm{~dB}$, which might be too low for some applications, especially those with amplitude modulation. Replacement of the header coupler with a circulator would increase the received signal power to $6 \mathrm{~dB}$, but it would also approximately double the final ASE level, yielding only a 3-dB improvement in the final SNR values.

To increase the values of SNR that are obtained with the single structure we propose the use of the doublebus topology presented in Fig. 1(b), ${ }^{4}$ in which the upper bus is built with erbium-doped fiber and the lower one uses standard fiber. In this case the return signal from the sensors is collected in the lower bus formed by couplers with a 5\% coupling ratio. We selected this low coupling ratio to avoid high losses in the nonamplifying lower bus. The results are presented in Fig. 3 and Table 1. In the double configuration the ASE noise level coming from the active fiber is highly attenuated when it passes through the 90:10 and the 95:5 couplers. Although the losses in the lower bus are not compensated for by active fiber segments, the signal power received from each channel at the monitoring point is very close (within $1 \mathrm{~dB}$ ) to that obtained in the single-bus topology. This fact is due to the absence of the 50:50 header coupler, which produces high losses in the single-bus topology. The reduction of the ASE level translates into an increment of $20 \mathrm{~dB}$ in the SNR values, which turn out to be greater than $32 \mathrm{~dB}$. We gave the first positions a slightly higher gain to minimize the effects of the greater attenuation suffered by the signals in the lower bus. After we apply the same allocation technique that we used in the single configuration, the power difference between channels has a maximum value of $1 \mathrm{~dB}$.

In conclusion, we have compared experimentally two different wavelength-division-multiplexed datagathering networks based on distributed optical amplifier buses. The improvement in the equalization among channels when distributed amplification is used has been confirmed. We have also experimentally shown the advantage in terms of signal-to-noise values of the double distributed optical amplifier fiber bus network versus the single-bus topology, as was predicted based on a previously reported theoretical result. ${ }^{4}$

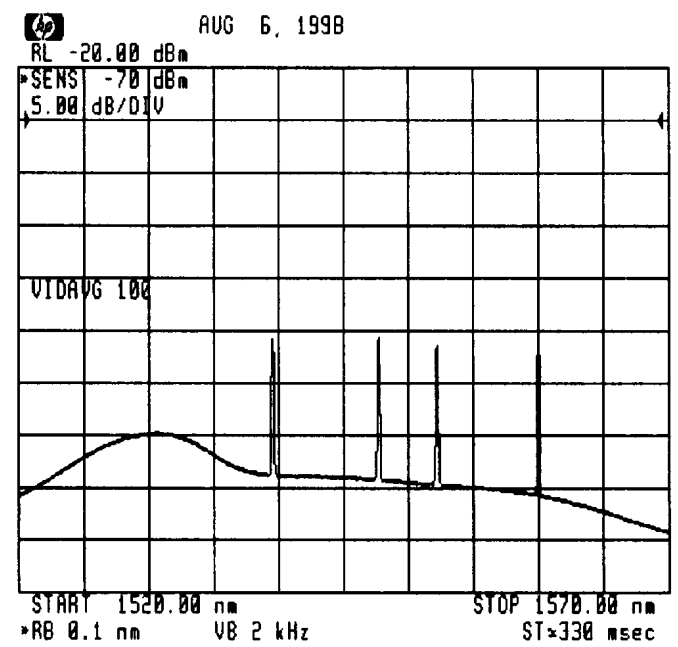

Fig. 2. Output power of the single distributed optical amplifier bus network, as measured by the spectrum analyzer.

Table 1. Signal Power, Noise Level, and SNR for the Single- and the Double-Bus Topologies

\begin{tabular}{ccccccccc}
\hline & \multicolumn{3}{c}{ Single Bus } & & \multicolumn{3}{c}{ Double Bus } \\
\cline { 2 - 3 } \cline { 6 - 8 } $\begin{array}{c}\text { Wavelength } \\
(\mathrm{nm})\end{array}$ & $\begin{array}{c}\text { Signal } \\
(\mathrm{dBm})\end{array}$ & $\begin{array}{c}\text { Noise } \\
(\mathrm{dBm})\end{array}$ & $\begin{array}{c}\text { SNR } \\
(\mathrm{dB})\end{array}$ & $\begin{array}{l}\text { Signal } \\
(\mathrm{dBm})\end{array}$ & $\begin{array}{c}\text { Noise } \\
(\mathrm{dBm})\end{array}$ & $\begin{array}{c}\text { SNR } \\
(\mathrm{dB})\end{array}$ \\
\hline 1539.60 & -41.0 & -53.7 & 12.7 & -41.7 & -74.0 & 32.3 \\
1547.77 & -40.9 & -54.2 & 13.3 & & -41.7 & -74.6 & 32.9 \\
1552.23 & -41.7 & -55.0 & 13.3 & & -41.9 & -74.9 & 33.0 \\
1560.09 & -42.6 & -55.6 & 13.0 & & -40.9 & -75.5 & 34.6 \\
\hline
\end{tabular}

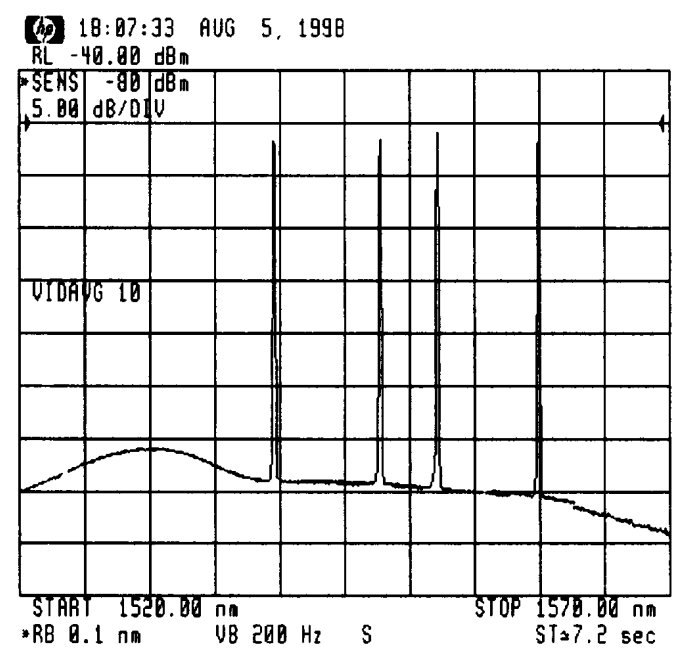

Fig. 3. Output power of the double distributed optical amplifier bus network, as measured by the spectrum analyzer. 
Financial support through Spanish Education Ministry projects TIC98-0397-C03 and TIC95-631-C04 and by the Gobierno de Navarra is acknowledged. We thank P. Urquhart, R. Hernandez, A. Loayssa, I. Matías, and F. Arregui for their help and discussion on this subject. M. Lopez-Amo's e-mail address is mla@upna.es.

\section{References}

1. J. L. Wagner, C. W. Hodgson, M. J. F. Digonnet, and H. J. Shaw, J. Lightwave Technol. 15, 1681 (1997).
2. B. Vizoso, I. Matías, M. López-Amo, and M. A. Muriel, Opt. Quantum Electron. 27, 847 (1995).

3. M. López-Amo, L. T. Blair, and P. Urquhart, Opt. Lett. 18, 1159 (1993).

4. R. Hernandez-Lorenzo, M. López-Amo, and P. Urquhart, J. Lightwave Technol. 16, 485 (1998).

5. P. Datta, I. Matías, C. Aramburu, A. Bakas, M. LópezAmo, and J. M. Otón, Microwave Opt. Technol. Lett. 11, 93 (1996). 